\title{
Linx
}

Revue des linguistes de l'université Paris X Nanterre

$10 \mid 1998$

L'indicible et ses marques dans l'énonciation

\section{Au cœur de l'indicible : le verbe dire}

Jean-Jacques Franckel

\section{(2) OpenEdition}

Journals

Édition électronique

URL : http://journals.openedition.org/linx/968

DOI : $10.4000 / \operatorname{lin} x .968$

ISSN : 2118-9692

\section{Éditeur}

Presses universitaires de Paris Nanterre

\section{Édition imprimée}

Date de publication : 1 juillet 1998

Pagination : 53-69

ISSN : 0246-8743

\section{Référence électronique}

Jean-Jacques Franckel, «Au cœur de l'indicible : le verbe dire », Linx [En ligne], 10 | 1998, mis en ligne

le 03 juillet 2012, consulté le 19 avril 2019. URL : http://journals.openedition.org/linx/968 ; DOI :

10.4000/linx.968

Ce document a été généré automatiquement le 19 avril 2019.

Département de Sciences du langage, Université Paris Ouest 


\title{
Au cœur de l'indicible : le verbe dire
}

\author{
Jean-Jacques Franckel
}

\section{Introduction}

1 Le verbe dire est, directement ou non au coeur de nombreux travaux, notamment dans le cadre de l'analyse du discours et de la paraphrase, de la grammaire textuelle, et des courants linguistiques qui traitent de l'énonciation. Dans cet article, ce verbe sera abordé dans une perspective de sémantique lexicale. Nous chercherons à en donner une caractérisation et à dégager une organisation dans la variation des sens qu'il peut prendre, en désintricant ce qui revient au verbe d'un côté et à ses entourages possibles de l'autre.

2 L'analyse proposée se fonde sur une double hypothèse ${ }^{1}$, censément applicable à toute unité lexicale :

- cette variation concerne les différents modes d'interaction possibles entre l'unité et son environnement ;

- cette interaction met en jeu d'une part des paramètres spécifiques au verbe, et d'autre part des principes d'organisation réguliers et généraux, indépendants de ces paramètres.

Dans cette perspective, ce qui peut se percevoir comme la "polysémie" de ce verbe en particulier et de toute unité lexicale en général résulte de la conjonction de différents plans de variation.

\section{Vers une caractérisation du verbe dire}

\subsection{Considérons la séquence}

(i) Paul me dit quelque chose

4 L'interprétation immédiate de cette séquence confère à Paul le statut d'une personne qui exprime et communique quelque chose par la parole. 

la reprise du sujet (Paul) par ça, qui a pour effet d'en modifier radicalement le statut :

\section{(ii) Paul, ça me dit quelque chose.}

7 Cette séquence signifie que la mention du nom de Paul évoque pour moi quelque chose, ou encore me rappelle quelque chose. Dans ce cas, le sujet syntaxique (désormais noté $\mathrm{C}_{\mathrm{o}}$ ) correspond non à une personne, mais à un événement: l'évocation du nom d'une personne. Le quelque chose en question n'a de statut qu'en tant que dit, il ne relève pas d'un à dire comme dans le cas de (i). On peut proposer la glose suivante : quand me est en relation à Paul, il y a chez / pour moi $(m e)$ un dit qui n'a pas d'existence indépendamment de l'actualisation de cette mise en relation et dont la détermination qualitative ne vient que de me : ça me dit ce que ça me dit. (ii) :

9 Dans le cas de (i) :

- la présence d'un complément attributif (en l'occurrence me) n'est pas obligatoire (Tais-toi donc! Paul dit quelque chose) ;

- on ne peut se passer de la présence d'un complément (noté $C_{1}$ ): le verbe implique la présence d'un objet minimal, inscrit dans le prédicat lui-même, traditionnellement appelé complément interne, et le rôle du $\mathrm{C} 1$ est de spécifier cet objet interne, à travers lequel du à dire se dit. Au C1 quelque chose peuvent se substituer toute une série de termes plus déterminés, pour peu qu'ils spécifient le complément interne : Paul me dit des bêtises, des mots d'amour, etc.

Dans le cas de (ii), ces critères s'appliquent à rebours :

- me est obligatoire ( ?Le nom de Paul dit quelque chose) ;

- quelque chose est le seul $\mathrm{C}_{1}$ possible de façon naturelle. Le dit n'est pas une actualisation d'un à dire dissocié du dit, il n'a pas d'autonomie par rapport au procès, et ne correspond pas à la spécification d'un objet interne.

11 Par ailleurs, on observe que dans d'autres types d'exemples, la présence du $\mathrm{C}_{1}$ se trouve bloquée : Viens donc chez moi, si ça te dit, le $C_{o}$ apparaissant directement ici sous une forme verbale : venir chez moi, repris par ça. Ici encore, on peut intégrer dire à un réseau de synonymes (tenter, faire plaisir) ${ }^{2}$ très éloignés des précédents.

Ces premiers exemples fournissent une première illustration de la variation du verbe. Celle-ci se traduit par l'apparition de sens divers, entre lesquels il n'existe pas de lien évident a priori.

Rien par exemple ne permet d'apparenter les deux sens de dire que manifeste l'ambiguïté d'une séquence comme Ça ne me dit rien (ça n'évoque rien / ça ne me tente pas). Ni de les associer à celui qui apparaît dans des exemples comme on dirait qu'il va pleuvoir, disons que je préfererais être en vacances, ou encore dites-donc, vous ne pourriez pas faire attention! ? La diversité des compléments possibles du verbe fait apparaître un autre aspect de la variation du verbe. Dire est associé à des sens bien différents dans dire la messe, dire une bêtise, dire un secret, pour s'en tenir à un échantillon limité. 

les propriétés du verbe pertinentes dans l'organisation de cette variation ne sont pas déductibles de quelque sens premier dont tous les autres pourraient être dérivés par analogie ou par métaphore. Un tel sens premier consisterait en un impossible compromis entre les deux contraintes antagonistes qui conduisent d'un côté à surinvestir sémantiquement le mot pour ne rien rater de sa spécificité et, de l'autre, à en abstraire un contenu suffisamment général pour permettre d'embrasser la totalité de ses emplois.

1.2. La caractérisation que nous proposons est conçue comme le schéma directeur que nous adopterons pour appréhender l'organisation de la variation du verbe ${ }^{3}$. Elle introduit des paramètres qui peuvent se trouver mobilisés de façon variable. Elle prendra la forme suivante, à la fois abstraite et minimaliste :

Dire établit un accès à un contenu qualitatif $\mathrm{P}$, en marquant l'inscription de $\mathbf{P}$ dans un champ subjectif ou intersubjectif sous forme de $p$. La notation $\mathrm{p}$ renvoie à $\mathrm{P}$ dit, ou encore $\mathrm{P}$ r
dans un champ subjectif ou intersubjectif. différentes figures possibles de la notion d'accès ${ }^{4}$. L'accès est lié à une orientation, éventuellement à une téléonomie: il y a orientation du non perceptible vers le perceptible.

Dire implique la mise en relation d'un ordre d'existence (le contenu qualitatif $\mathrm{P}$ ) qui n'est pas matérialisable / communicable / exhibable / perceptible en soi, à une trace de cette existence, et qui n'en est jamais qu'une trace, que nous notons $p$. Cette trace, cette réalisation particulière $\mathrm{p}$ de $\mathrm{P}$ rendue accessible à un ou des sujet(s) est ce que nous appelons une occurrence de $\mathrm{P}^{5}$. La spécificité du verbe dire tient à ce que cette occurrence est construite relativement à un champ subjectif ou intersubjectif. Le sujet syntaxique $\mathrm{C}_{\mathrm{o}}$ du verbe dire correspond à l'instance qui fait passer de $\mathrm{P}$ à $\mathrm{p}$. Elle peut être ou non de nature subjective. Ainsi, dans le cas de cette histoire me dit quelque chose, $\mathrm{C}_{\mathrm{o}}$ qui correspond à cette histoire n'est pas de nature subjective, contrairement à ce qui est le cas dans un exemple comme Paul m'a dit une chose étonnante. Le premier exemple signifie que un contenu qualitatif $(\mathrm{P})$ est rendu accessible au sujet auquel réfère $m e$, et que ce contenu qualitatif se présente sous la forme de quelque chose (p). Le second exemple signifie que Paul m'a rendu accessible un contenu qualitatif $\mathrm{P}$ sous la forme de une chose étonnante.

\section{Variation du verbe}

21 Nous considérerons deux ordres de variation.

22 2.1. La première concerne le mode d'interaction du verbe avec d'autres termes de l'entourage du verbe.

$23 *$ Dans un premier cas (A), seul le verbe dire introduit la problématique de l'accès. Dire construit une "mise en dire" d'un contenu P qui n'a aucun statut indépendamment de ce dire.

Dire ne fait pas que poser un accès en soi, il est associé à la construction même de l'entité $\mathrm{P}$ qu'il rend accessible. L'instance de construction de P n'est pas de nature subjective. 
\begin{tabular}{|l|}
\hline Jean, ça me dit quelque chose \\
\hline Quelque chose me dit que ça va mal tourner \\
\hline Un apéritif, ça te dit?
\end{tabular}

Dans ces exemples, que nous analyserons ci-après, $\mathrm{P}$ n'est pas construit préalablement au fait d'être rendu accessible : il n'est pas construit comme à dire, ou du moins n'est à dire que dans la mesure ou il est dit. L'accès est constitutif de ce à quoi il donne accès.

* Dans un deuxième cas (B), $\mathrm{P}$ est construit comme à dire indépendamment du fait d'être dit. Il y a dissociation de $\mathrm{P} \grave{a}$ dire et de $\mathrm{P}$ dit. Dire fait passer de $\mathrm{P}$ à dire à $\mathrm{p}$, il rend perceptible et partageable $\mathrm{P}$ à un ou à d'autres sujets sous forme de $\mathrm{p}$ qui correspond alors à une actualisation donnée parmi d'autres possibles de $\mathrm{P}$ à dire : un rapport variable s'établit entre du à dire et du dit. Le rapport de $\mathrm{p}$ à $\mathrm{P}$ correspond à des ajustements variables.

28 Par exemple dans dire la messe, la messe est déterminée comme à dire, et il y a stricte coïncidence entre le à dire et le dit; dans dire oui, oui est contextuellement déterminé comme une réponse, en elle-même inscrite dans la problématique du à dire.

Il y a intrication entre le verbe dire et un support de à dire, constitué de façon privilégiée (mais non exclusive) par le complément direct du verbe.

30 * Dans un troisième cas enfin (C), il y a d'un côté indépendance et de l'autre interaction entre dire et des éléments de son environnement. D'un côté, dire est mis en jeu indépendamment d'un à dire. De l'autre, des éléments de l'entourage fournissent le support d'un à dire, dès lors que du dit est construit (les grenouilles coassent, ça veut dire qu'il va pleuvoir).

31 Ce premier mode de variation met donc en jeu trois configurations générales d'articulation d'une unité avec son entourage

2.2. Le deuxième ordre de variation (correspondant aux cas notés 1., 2. et 3.) concerne le mode de constitution de la mise en relation entre $\mathrm{P}$ et $\mathrm{p}$. L'accès est pris en compte à partir de $\mathrm{P}$ (cas 1.), de $\mathrm{p}$ (cas 2.) ou de $\mathrm{P}$ et $\mathrm{p}$ à la fois (cas 3.).

33 Ce deuxième ordre de variation est en prise directe sur les paramètres constitutifs de la caractérisation du verbe.

Cas A. Il n'y a pas de support de à dire indépendamment du verbe dire et de l'actualisation d'un dit. L'instance de construction de P n'est pas de nature subjective.

C'est la construction même de $\mathrm{P}$ opérée dans le temps à travers l'actualisation de Co qui rend $\mathrm{P}$ accessible à un sujet sous forme de $\mathrm{p}$. A travers cette construction, $\mathrm{P}$ n'a pas de contenu qualitatif en soi : il est "imperceptible", il n'est pas à dire, c'est en lui-même un contenu vide ("quelque chose") : c'est $\mathrm{p}$ qui lui confère son contenu qualitatif, $\mathrm{p}$ étant construit par une instance subjective. Ce que l'on peut représenter par le schéma : 


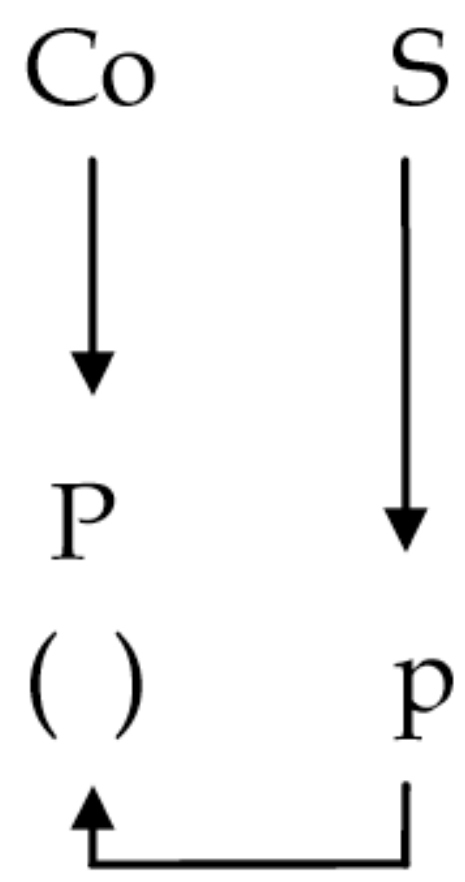
et $\mathrm{p}$ cas (1., 2. et 3.) que nous énonçons brièvement pour fixer les idées, avant de les reprendre dans le détail.

L'actualisation de Co construit $\mathrm{P}$ comme non perceptible (figuré par des parenthèses vides) en tant que dépourvu de tout contenu qualitatif, et dire marque le passage à travers cette construction même de $\mathrm{P}$, non perceptible, à p perceptible sous forme d'un contenu qualitatif donné.

Le complément du verbe dire confère à $\mathrm{p}$ une présence matérielle dans l'énoncé, par exemple quelque chose, ou que ça va mal tourner. En revanche, P n'a aucune présence matérielle et ne peut être nommé (c'est un contenu vide), si ce n'est justement par le truchement du contenu qualitatif que lui confère $p$.

Le rapport de $\mathrm{p}$ à $\mathrm{P}$ n'est pas de l'ordre d'un ajustement, mais d'un investissement. Le dit n'est pas une façon de dire, c'est la manifestation pour et par un sujet d'un contenu à la fois existant indépendamment de lui et imperceptible en tant que dépourvu de tout contenu qualitatif propre. D'un côté, $\mathrm{P}$ n'a d'autre détermination qualitative que celle que lui confère $p$. D'un autre côté, $p$ n'est construit par un sujet que dans la mesure où il y a construction de $\mathrm{P}$ dans le plan temporel, et hors du plan subjectif.

9 Ce premier cas se trouve croisé par les trois modes de constitution de la relation entre $\mathrm{P}$ represtail.

$$
\begin{array}{|l|l|}
\hline \text { - A.1 } \\
\hline \text { Ex. Jean, ça me dit quelque chose. } \\
\hline
\end{array}
$$

40 On part de la construction de $\mathrm{P}$ à travers l'actualisation de Co, et cette construction déclenche du même coup son investissement par un contenu qualitatif p. P n'a d'autre contenu que celui que lui confère $\mathrm{p}$ qui, de son côté n'a de statut que relativement à la 
construction de P par Co. C'est ici l'accès lui-même qui est constitutif d'un contenu, en tant que l'accès est toujours accès à quelque chose.

- A.2.

Quelque chose me dit que ça va mal tourner

41 A partir d'un contenu qualitatif déterminé $\mathrm{p}$, dire marque que cette construction constitue un accès à $P$ construit à travers l'actualisation de Co.

- A.3.

Un apéritif, ça te dit?

On part des deux constructions à la fois, à travers une relation intersubjective.

Nous reprenons ci-dessous de façon plus détaillée l'examen de ces différents cas.

\begin{tabular}{|l|l|}
\hline A.1. \\
\hline Ce visage me dit $q c h$ \\
\hline Jean, ça me dit qch. \\
\hline
\end{tabular}

Co est préconstruit. Il fait l'objet d'une reprise : Jean est repris par ça. Il s'agit de l'évocation de Jean, et l'énoncé est facilement précédé de termes comme tiens!, ah oui!, etc.. Co déclenche la construction d'un contenu imperceptible ( $\mathrm{P}$ vide de tout contenu qualitatif propre), et dire marque que cette construction de $\mathrm{P}$ est indissociable du fait que $\mathrm{P}$ est rendu perceptible. La construction de $\mathrm{P}$ comme non perceptible s'opère dans le même mouvement que le processus qui le rend perceptible sous forme de $\mathrm{p}$.

Le contenu qualitatif $\mathrm{p}$ se réduit au seul fait de l'existence de $\mathrm{P}$ rendu perceptible à un sujet à travers sa construction même. Ce contenu est rendu existant pour le sujet auquel réfère $m e$, mais lui échappe : $\mathrm{p}$ est réduit à quelque chose, ce qui signifie que le contenu de $\mathrm{p}$ revient dès lors à la seule propriété d'être perceptible par le sujet auquel réfère me. Il y a construction de perceptible à partir de l'actualisation de Co. Ni P ni $\mathrm{p}$ ne sont définissables. L'accès que $p$ confère à $P$ se réduit au seul fait de sa construction et est indépendant de tout contenu qualitatif singulier. On ne peut dans le même temps dire que quelque chose advient à l'existence et ce qu'est ce quelque chose.

Il y a donc une positivité purement existentielle et non qualitative de $\mathrm{P}$ à travers l'actualisation de Co. Dire marque ici le seul passage de non perceptible à perceptible de $\mathrm{P}$ en un site singulier me. A travers Jean, un processus de perception se met en place, mais les entités mises en relation ne sont pas premières par rapport à cette mise en relation, et n'ont d'autre contenu que le fait même d'être mises en relation. 
A.2.

Quelque chose me dit que ça va mal tourner.

On part cette fois d'un contenu qualitatif déterminé $\mathrm{p}$ (ça va mal tourner). Comme le marque l'introduction de $\mathrm{p}$ par une complétive, dire marque que si me perçoit $\mathrm{p}$, c'est que $\mathrm{p}$ correspond à un contenu $\mathrm{P}$ rendu perceptible, $\mathrm{P}$ étant construit par la prédication d'existence de quelque chose. Il y a "mise en dire" d'un contenu (P) qui ne se manifeste (p) qu'à travers cette mise en dire.

Le sujet correspondant à me pose ça va mal tourner, et établit en même temps que cet état de chose $\mathrm{p}$ lui est rendu perceptible par l'actualisation dans le temps d'un déclencheur qui reconstitue ce perceptible comme issu de la prédication d'existence d'une entité non perceptible $\mathrm{P}$ qui est révélée (à $m e$ ).

Cette fois, Co n'est pas préconstruit, il fait l'objet d'une prédication d'existence. Le fait que $\mathrm{p}$ soit perceptible est lié à la prédication d'existence de Co et de $\mathrm{P}$ dans le temps. L'actualisation de Co est à la fois condition et conséquence du passage à $\mathrm{p}$ : elle est à la fois seconde dans la mesure où l'on part de $\mathrm{p}$ du point de vue du mode de constitution de la relation, et première dans la mesure où $\mathrm{p}$ est de l'ordre du perceptible, second par rapport au non perceptible. Co est nécessairement indéterminé : sa détermination se réduit au seul fait qu'il s'actualise. Co n'a lui-même d'autre détermination que celui de déclencheur du passage de non perceptible à perceptible. On ne peut avoir d'autre sujet ici que quelque chose.

\section{A.3. Double mode de constitution de la relation. On part à la fois de $\mathbf{P}$ et de $\mathbf{p}$.}

\section{Un apéro, ça te dit?}

Dire entretient une parenté avec avec le verbe tenter ${ }^{6}$, Ce type d'emploi se présente nécessairement sous la forme de question et correspond à une proposition. Il y a absence de tout complément direct, présence nécessaire d'un complément attributif. Enfin, Co fait nécessairement l'objet d'une reprise (ça).

3 Un apéro construit par Co est-il de l'ordre du dit pour te ? La question porte sur l'existence même d'un accès venant de toi à ce qui est construit par la mention de apéro par moi. Dire marque l'inscription dans l'ordre d'un dit par te d'une proposition (un apéro) introduite par l'énonciateur.

54 A travers la forme ici obligatoire de la question, on dissocie deux constructions de $\mathrm{p}: \mathrm{p}$ en tant que dit / non dit par te, et $\mathrm{p}$ en tant que dit par le locuteur: $\mathrm{p}$ est donc en préconstruit par celui qui pose la question, ce qui relève du fonctionnement de type 2., en même temps que pour le sujet sollicité, c'est $P$ qui est premier, ce qui relève du type 1 . Ce qui est d'emblée un dit pour le locuteur (cas 2.) reste à constituer par te comme relevant (ou non) de l'ordre du dit (cas 1.).

\section{Conclusion sur le cas A.}

Dans le cas A, ce qui est en jeu, c'est la construction même d'une entité comme perceptible à travers l'actualisation de Co. Dire ne fait pas que poser un accès en soi, il est associé à la construction même de l'entité qu'il rend perceptible, sans que celle-ci soit nécessairement première par rapport à cet accès. La construction de $\mathrm{P}$ ne l'investit jamais d'un contenu qualitatif propre 

sujet.
Dans le cas A.1, P est rendu perceptible à travers sa construction même par l'actualisation de Co, p n'ayant d'autre détermination qualitative que le fait même d'être perceptible.

Dans le cas A.2., p est premier, et l'on reconstitue $\mathrm{p}$ à la fois comme ce qui résulte de la construction de $\mathrm{P}$ à travers l'actualisation de $\mathrm{Co}$, et comme ce qui donne accès à $\mathrm{P}$.

Dans le cas A.3., on part à la fois de $\mathrm{P}$ d'un côté, et de $\mathrm{p}$ de l'autre, à travers une dimension inter-subjective.

Cas B. Il y a support de à dire indépendamment du verbe dire. $\mathrm{P}$ est construit par un

$\mathrm{P}$ est muni d'un contenu qualitatif indépendamment de l'accès qu'en fournit $\mathrm{p}$. Ce contenu est construit par un sujet en tant que destiné à être perceptible (à dire). La construction de P n'est plus de l'ordre d'une actualisation de Co dans le temps, elle relève d'une instance subjective, hors du plan temporel. Dire rend perceptible ce contenu singulier $\mathrm{P}$ à un ou à d'autres sujets sous forme de $\mathrm{p}(\mathrm{P}$ dit) qui devient dès lors de l'ordre $\mathrm{du}$ partageable. Dire fait passer de à dire à dit, et $\mathrm{p}$ correspond alors à une actualisation donnée, ou encore une occurrence singulière de $\mathrm{P}$ à dire. $\mathrm{P}$ et $\mathrm{p}$ s'inscrivent dans des rapports variables, relevant d'ajustements variables. En d'autres termes, il peut y avoir un zonage du à dire. Le dit est spécification d'un à dire ("disible"). C'est la façon dont le à dire s'actualise, le dit est une façon de dire: pi est articulable, dans des jeux d'altérité variables à d'autres façons de dire, pj, pk.

Le cas B se trouve de nouveau croisé par les trois modes (1., 2. et 3.) de constitution de la mise en relation entre $p$ et $P$ ).

\section{B1. La relation se constitue ^ partir de $P$.}

P se présente muni d'un contenu propre et indépendant de $\mathrm{p}$ qui n'en constitue qu'une actualisation. Ce cas concerne l'emploi de dire avec des compléments bien spécifiques : il s'agit de termes tels que la messe, les quatre vérités, l'avenir (seul un médium dit l'avenir), son fait à quelqu'un qui se présentent intrinsèquement comme supports de à dire. Dans ce cas, $\mathrm{p}$ ne constitue pas une division ou un zonage du à dire, il n'en constitue qu'une actualisation. La singularité du sujet ne joue pas.

\section{B2. La relation se constitue à partir de $\mathbf{p}$.}

L'accès est envisagé du point de vue de $\mathrm{p}: \mathrm{p}$ correspond à la reprise d'un contenu (noté $\pi$ ) déterminé indépendamment de $P$ et de $p$, et qui n'est pas en soi de l'ordre d'un dire. On a affaire à à une "mise en dire" de $p$ qui, à travers $p$, donne un accès à $P$.

On a dans ce cas une décomposition $\mathrm{p} \mathrm{--->p--->P}$

Ainsi, dans un exemple comme Je vais te dire ce qui s'est passé, p correspond à l'événement ce qui s'est passé qui se trouve dit : ce qui s'est passé en tant que dit correspond à $\mathrm{p}$, ce qui par là même constitue $\pi$ comme à dire $(\mathrm{P})$. $\mathrm{p}$ n'est ni dit ni à dire, c'est le verbe dire qui intègre $\pi$ dans la problématique d'un rapport dit -à dire.

Nous analysons ci-dessous différents exemples relevant de ce type de construction. 
correspond dans ce cas à un premier dit qui se trouve reconstruit comme à dire $\mathrm{P}$, te que $\mathrm{p}$ (je viens) constitue un accès à ce à dire. On pose $\mathrm{p}$ comme étant un dit-à dire. Il peut s'agir d'une réponse à une question du type : Qu'est-ce qu'il t'a dit exactement ?).

Relèvent de la même construction des exemples comme La vérité je vais vous la dire! (Zola), ou encore Je vais te dire une chose: "p". On part de une chose ou de la vérité, et, en la disant, on la pose comme à dire.

- J'ai dit une bêtise.

Ce n'est pas une bêtise qui est à dire. On part d'un dit ( $\mathrm{p}$ : une bêtise), qui est une façon de rendre perceptible / partageable un à dire. Du fait des propriétés de bêtise, p est une façon de dire (pi) qui s'inscrit dans une relation d'altérité forte à d'autres façons de dire (de rendre $\mathrm{P}$ accessible), en particulier pj comme façon de dire adéquate. $\mathrm{P}$ n'est à dire que dans la mesure où il est dit (sous forme de une bêtise).

- Chut! Paul me dit quelque chose. Ici encore, ce n'est pas quelque chose qui est à dire. Quelque chose n'est qu'une façon (défectueuse) de constituer un accès au à dire. On part d'un dit qui n'est déterminé qu'en tant que perçu, rendu accessible, et ce quelque chose est une actualisation d'un à dire dont il constitue une occurrence. C'est le dit qui marque que quelque chose est à dire (mise en dire de quelque chose à travers ce dit).

- Je dis la vérité : je pose un contenu p qui est un dit tel que ce dit constitue un accès à P. C'est le dit qui est premier; on ne pose pas d'abord la vérité comme à dire, on la dit, ce qui suppose dès lors que ce quelque chose $(\mathrm{P})$ qui se dit comme vérité est à dire. Dans ce cas, on est dans une problématique de la suspicion ou du mensonge, du fait même que la vérité prend le statut d'un dit (p), qui se trouve par conséquent relativisé en tant que déterminé par un sujet, et ne constituant donc qu'un accès parmi d'autres à la vérité $\mathrm{P}$ comme à dire (c'est ma vérité, telle que je la dis qui donne accès à la vérité hors subjectivité).

On notera que le mode d'accès auquel correspond le verbe dire relève d'une discontinuité. Il s'agit d'un accès "global" et non séquenciel : on ne dit pas une histoire, on la raconte.

Il n'y a ni préconstruction de $\mathrm{P}$ préalablement à son accès, ni de $\mathrm{p}$ par rapport à ce dont $\mathrm{i}$ constitue un accès, ni $\mathrm{P}$ ni $\mathrm{p}$ n'est premier par rapport à l'autre. P-p reprennent en bloc un contenu $\pi$ construit indépendamment du dire et dire fait entrer $\mathrm{p}$ dans le champ d'un $\grave{a}$ dire-dit.

Cette reprise de $\mathrm{p}$ à travers une mise en dire de $\mathrm{p}$ peut prendre différentes formes :

- Emplois du type Il pleut, dit-il. 
79 Cette "mise en dire" de $\mathrm{p}$ met en jeu l'inversion et la postposition, par laquelle dire croise ici une série d'autres verbes partageant cette construction: crier, s'écrier, s'exclamer, soupirer, penser, etc.

\begin{tabular}{|l|l|}
\hline Il n'est pas toujours facile, je te le dis! \\
\hline - Il est complètement fauché, comme on dit. \\
\hline - Que tu dis!
\end{tabular}

80 Un contenu p est repris comme relevant de l'ordre d'un à dire-dit. Ce dit a une source (tu) inscrite dans une relation intersubjective où dit par toi se trouve confronté à dit par tout autre locuteur.

- Bon, je prends celui là, disons.

81 La postposition autonomise $p$ par rapport à $p$

- Il m'a dit de ranger la salle

Pourquoi ranges-tu la salle? Parce qu'il m'a dit de le faire.

82 Il faut tenir compte de la fonction de de. ranger la salle n'est pas a priori de l'ordre d'un dit. C'est dire qui inscrit ranger la salle comme relevant d'un dire.

- On y va. Aussitôt dit, aussitôt fait

83 Dire confère à on y va le statut d'un à dire qui se dit et ce dit se trouve redéployé comme à faire.

Eh bien, dites moi! Il a grandi !

Non mais dites, vous pourriez pas faire attention, non?

On le voit arriver sur scène, et tout est dit.

$84 \quad$ P et $p$ sont construits en bloc

85 Le contenu de $\mathrm{P}$ se confond ici avec celui de $\mathrm{p}$, ce contenu correspondant à une reprise de p.

86 Il n'y a pas division du à dire : p n'est pas une occurrence singulière de à dire, il en est la manifestation hors altérité. 

de son environnement.

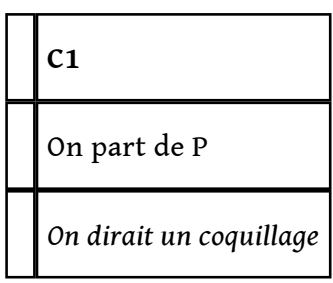

Cet énoncé correspond à la description d'un objet inconnu ou dont on sait qu'il n'est pas un coquillage.

Coquillage a à la fois le statut de $\mathrm{p}$ et le statut de $\mathrm{p}$. Un objet du monde, correspondant à un contenu $\mathrm{p}$ hors de la problématique du dire est constitué d'un côté comme à identifier, et de l'autre comme un à-dire-dit.

Comme dans le cas $\mathrm{A}$, dire correspond à la construction même d'un contenu qualitatif $\mathrm{P}$ sans contenu autre que celui auquel est conféré un accès sous la forme de coquillage qui l'investit de son contenu. Seul le verbe dire définit la problématique de l'accès.

Comme dans le cas B, $\mathrm{p}$ en tant que correspondant à un objet à identifier peut devenir le support d'un à dire dès lors que du dit est construit.

D'autre part, poser être coquillage à la fois comme ce qu'est un objet et ce qu'on en dit est nécessairement source d'une discordance. Il s'agit de déterminer coquillage comme accès à ce qu'est un objet donné tout en marquant qu'il ne s'agit que d'un accès et non d'une pure et simple identification. D'où l'emploi nécessaire du conditionnel qui permet de surmonter la contradiction de ces deux statuts de $\mathrm{p}$. La forme On dit que $\mathrm{p}$ correspondrait à un processus de dénomination ou à un décret

93 La présence de on revient à effacer la singularité de l'instance subjective de détermination de $\mathrm{p}^{7}$. Du même coup, $\mathrm{p}$ ne se situe pas dans une relation d'altérité à d'autres dits $\mathrm{pj}$ possibles.

94 L'accès est pris en compte à partir de $\mathrm{P}$ du fait que la mise en dire est opérée à partir d'un objet à identifier contextuellement déterminé. Il s'agit de la construction de type 1 .

$$
\begin{aligned}
& \text { C2 } \\
& \text { Dire qu'il était si mignon! }
\end{aligned}
$$

Ici encore, il était si mignon a un double statut. Il correspond d'un côté à p (il s'agit d'un constat, d'un savoir, qui du fait de la présence de si et de l'imparfait correspond au fait qu'il n'est plus du tout mignon) et à $p$ en tant que produit de l'inscription de $p$ dans le champ d'un dire. Dire n'est pas lié à la singularité d'un sujet (l'infinitif marque l'absence d'un sujet singulier).

- D'un côté, $\mathrm{p}$ entre dans le champ d'un à dire qui se dit, ce qui relève de A ;

- De l'autre, $p$ correspond à un constat qui, dès lors qu'il entre dans l'ordre du dire devient le support d'un à dire. 
loi marque une discordance et une collusion (à laquelle correspond l'exclamative) entre un savoir sur un état de choses $\mathrm{p}$ (il était si mignon en tant qu'impliquant que il n'est plus du tout mignon), et cet état de chose en tant que dit.

C3

Les grenouilles coassent, ça veut dire qu'il va pleuvoir.

Dans ce cas, on a une double préconstruction, et l'accès est pris en compte à la fois à partir de $\mathrm{p}$ et à partir de $\mathrm{P}$ :

- préconstruction de Co, qui fait l'objet d'une reprise (ça) d'une part ;

- préconstruction de p qui correspond à une complétive, d'autre part.

D'un côté, dire est mis en jeu indépendamment de toute problématique externe du dire. De l'autre, Co est interprétable comme un signe ou un signal et devient ainsi support potentiel d'un à dire.

La discordance entre le fait que c'est l'actualisation de $C_{o}$ qui déclenche une mise en dire d'un contenu nécessairement indéterminé et le caractère déterminé de $\mathrm{p}$ se traduit par le fait que $\mathrm{p}$ ne peut être dit effectivement. On en est réduit à la tension du non perceptible vers le perceptible, que marque la présence de vouloir ${ }^{8}$. Les grenouilles coassantes ne disent rien en elles-mêmes.

\section{Conclusion}

L'étymologie du verbe dire fait apparaitre la racine indo-européenne deik, dik, qui renvoie à l'acte de montrer, de désigner. Or montrer, sans le recours au contact et au toucher est une conduite spécifiquement humaine. Montrer implique une distance entre ce qui est montré et le sujet à qui l'on montre.

De même, dire est indissociable de l'indicible auquel il donne accès, mais dont il ne constitue jamais qu'un accès. Dire marque un accès à ce que l'on ne peut atteindre.

Le dit a été analysé comme constitutif d'un accès à un contenu qualitatif. La notion d'accès est liée à celle de téléonomie et, de ce point de vue, dire peut être associé à un vouloir dire. La mise en jeu du fonctionnement du verbe dire entraine la prise en compte de trois formes de vouloir dire : le vouloir dire du monde, le vouloir dire du sujet et le vouloir dire des mots.

S'agissant du vouloir dire du monde, le contenu qualitatif auquel dire donne un accès n'est pas par lui-même de l'ordre d'un à dire, il n'est à dire et n'a de statut qu'en tant qu'il est dit. Il n'y a pas adéquation du dire au vouloir dire, le dit est constitutif de la détermination qualitative du contenu ainsi "mis en dire".

S'agissant du vouloir dire d'un sujet, le contenu qualitatif tel qu'il est dit par un sujet s'inscrit en revanche dans une problématique de l'adéquation / discordance : le dit est un accès plus ou moins adéquat au vouloir dire

6 Le vouloir dire des mots et des signes se caractérise enfin par le fait même que le dit ne peut se détacher du vouloir : les mots ne se comportent comme tels que lorsqu'ils veulent 
dire : le propre des mots est de dire et de ne dire - très exactement et sans échappatoire possible, sauf à les détourner et à les altérer en les remplaçant par d'autres mots - que ce qu'ils veulent dire.

\section{BIBLIOGRAPHIE}

AUTHIER-REVUZ J. (1995) Ces mots qui ne vont pas de soi. Boucles réflexives et non coïncidence du dire, collection Sciences du langage, Larousse.

CULIOLI A.(1997) Accès et obstacles dans l'ajustement intersubjectif, in Mélanges Jean-Blaise Grize, Mieville, Berendonner eds, Peter Lang, p. 239-248.

CULIOLI A. (1990) Pour une linguistique de l'énonciation, tome 1, Ophrys

DE VOGÜÉ S. (1993) Des temps et des modes, in Le Gré des langues, nº 6, L'Harmattan, p. 65- 80

\section{NOTES}

1. Dans son fondement, cette hypothèse s'inscrit dans un programme de recherche "identité et variation lexicale" actuellement développé au sein de l'unité de recherce URA 1028 associée à l'université de Paris7.

2. Relevons le cas du verbe chanter, qui peut facilement se substituer à dire dans ce cas : Vas-y, si ça te chante! Toutefois, il existe une différence sensible entre si ça te dit et si ça te chante. Si ça te dit est incitatif, relève d'une invite, alors que si ça te chante renvoie à une désolidarisation de l'énonciateur vis à vis d'une décision qu'il désapprouve.

Cet exemple montre que chanter fait apparaître quoique avec des contraintes très différentes, des phénomènes du même ordre que dire. On a d'un côté des exemples comparables à (1): Jean chante une chanson, où Jean est, en la circonstance, un chanteur et où chanson qualifie le chantable inscrit en tant qu'objet interne dans le prédicat lui-même.

3. Pour les besoins de la présentation, nous prenons le parti de ne pas justifier cette caractérisation a priori. Elaborée à partir des exemples présentés dans la suite de l'article, c'est l'analyse même de ces exemples qui l'étayera au fur et à mesure.

4. L'accès prend le statut d'un concept actuellement travaillé par A. Culioli. Cf; en particulier Culioli (1997)

5. Cette mise en jeu du terme d'occurrence peut s'inscrire directement dans le cadre de la théorie d'Antoine Culioli sur les domaines notionnels. Sur ce point, cf. Culioli (1991) et S. De Vogüé (1993), p. 68-75

6. mais aussi de façon plus immédiatement illustrative de la caractérisation de dire, avec le verbe brancher : un apéro, ça te branche?

7. Il convient de distinguer cet exemple d'un exemple comme Je dirais plutôt que c'est un coquillage, où $\mathrm{p}$ s'inscrit en revanche dans une altérité marquée à d'autres accès $\mathrm{pj}$, pk possibles à $\mathrm{P}$.

8. vouloir : étant donné $\mathrm{P}$ envisagé (visé), vouloir marque que $\mathrm{P}$ effectif / actualisé ne dépend pas du constructeur de $P$ envisagé.

Vouloir permet la mise en jeu de trois points de vue relativement à ce hiatus.

$1^{\circ}$ Le point de vue du constructeur de P envisagé : Il veut y aller, je veux lui parler 
$2^{\circ}$ Le point de vue de qui est en mesure de satisfaire et de réaliser l'actualisation de P. Il s'agit d'un emploi intersubjectif. Avec l'imparfait, on peut avoir simultanément les deux points de vue : Et le monsieur, qu'est-ce qu'il voulait?

$3^{\circ}$ Le point de vue de ce qui est visé : Le sommeil ne veut pas venir. Etant donné $\mathrm{P}$ visé, $\mathrm{P}$ ne s'actualise pas

On peut distinguer trois types fondamentaux de vouloir dire: le vouloir dire du monde (les grenouilles coassent, ça veut dire qu'il va pleuvoir); le vouloir dire d'un sujet (tu vois ce que je veux dire ?) ; le vouloir dire des mots (ils signifient).

\section{RÉSUMÉS}

Cet article vise à dégager ce qui est propre à fonder l'unité du verbe dire en français à travers la diversité de ses emplois et de ses constructions.

This paper aims at bringing out the characteristics of the French verb dire liable to found its identity through the diversity of its various uses and constructions.

\section{AUTEUR}

JEAN-JACQUES FRANCKEL

Université de Paris X - Nanterre 